Sanja Radanović ${ }^{*}$

Universität Banja Luka
UDK 323.15(497.15=112.2)"1878/1914"

DOI: 10.4312/linguistica.60.2.213-225

\title{
DEUTSCHE KOLONIEN IN BOSNIEN-HERZEGOWINA BIS 1914
}

\section{EINLEITUNG}

Nach der Okkupation Bosnien-Herzegowinas durch die österreichisch-ungarische Monarchie 1878 folgte ein bedeutender Zufluss von Ausländern. Einerseits waren das Ansiedler, die ins Land gebracht wurden, um Angestellten- und Beamtenposten in der neugegründeten Administration zu bekleiden, die eine Grundlage des österreichischungarischen Regimes bildete (Preradović u. a. 2017: 13) und andererseits war das eine hohe Anzahl an Bauern, die systematisch angesiedelt wurden oder die durch private Initiative kamen.

Zum Zeitpunkt der Okkupation war Bosnien-Herzegowina ein äußerst unterentwickeltes Land. Es herrschten feudale Verhältnisse mit muslimischen Großgrundbesitzern und armen freien und abhängigen Bauern, die am zahlreichsten waren. Das bedeutet, dass die meisten Einwohner auf dem Land lebten. Weitere Merkmale des Landes waren eine Naturalwirtschaft, eine extensive Landwirtschaft, die Nichtexistenz von Industrie, der Mangel an Straßen und ein niedriges Bildungsniveau (Preradović u. a. 2017: 13). Dementsprechend stellte jede Neuigkeit, die Österreich-Ungarn einführte, einen bestimmten Fortschritt in der Entwicklung von Bosnien-Herzegowina dar. Dazu trugen in einem gewissen Maße auch nationale Minderheiten, besonders die deutsche, bei.

Im vorliegenden Beitrag werden Mitglieder der deutschen nationalen Minderheit, genauer gesagt deutsche Bauern, während der österreichisch-ungarischen Regierung in Bosnien-Herzegowina, d. h. von der Ankunft der ersten Ansiedler in den letzten Jahrzehnten des 19. Jahrhunderts bis Anfang des Ersten Weltkrieges, dargestellt. Der besondere Schwerpunkt wird auf die deutschen Kolonien im nordwestlichen Teil Bosnien-Herzegowinas gelegt, die gleichzeitig die größten und reichsten Kolonien waren, und zwar auf die historische Entwicklung dieser Kolonien, auf die Art, das Leben zu organisieren, sowie auf die Schulangelegenheiten in diesen Kolonien. Kurz werden auch andere deutsche Kolonien in Bosnien-Herzegowina dargestellt, um zu sehen, in welchen Teilen von Bosnien-Herzegowina angesiedelte deutsche Bauern lebten, wie sie ihr Leben in der neuen Heimat organisierten und was ihr Kommen für BosnienHerzegowina bedeutete. Hier werden die Deutschen nicht berücksichtigt, die sich in bosnisch-herzegowinischen Städten ansiedelten, da sie zu einem anderen sozialen Umfeld gehörten und keine kompakten Gemeinschaften bildeten wie angesiedelte Bauern, weswegen es zugänglichen Quellen zufolge sehr schwer ist, deren Anzahl sowie deren Positionen in der bosnisch-herzegowinischen Gesellschaft festzustellen.

sanja.radanovic@flf.unibl.org 


\section{DEUTSCHE ANSIEDLER IN BOSNIEN-HERZEGOWINA}

Die ersten Ansiedler vom deutschsprachigen Gebiet kamen schon im Mittelalter nach Bosnien-Herzegowina. Das waren sächsische Bergleute, die dank ihrer erfolgreichen Arbeit in ganz Europa geschätzt wurden. Im 11. Jahrhundert kamen sie auf den Balkan, auch nach Bosnien, wo sie Bergwerke eröffneten. In Bosnien übernahmen sie fast alle Bergwerke. Hier hielten sie sich bis zur Ankunft der Türken im 15. Jahrhundert auf, als die Erzgewinnung plötzlich sank. Auch das, was übrigblieb, wurde nach den Vorschriften und Verfahren der sächsischen Bergleute geführt. Später bildeten die Türken ein Gesetz, welches sie das sächsische Gesetz (Kanun Sas) nannten. Von der Tätigkeit der sächsischen Bergleute in Bosnien, besonders im östlichen Teil, zeugen auch einige Toponyme, z. B. Dörfer mit den Namen Sas und Sasina (Richembergh 2010: 31, 32).

Während der osmanischen Regierung war die Anzahl der Deutschen in BosnienHerzegowina sehr niedrig, es gab nur ein paar Familien und Einzelpersonen (Zaplata 1934: 919).

Das nächste bedeutende Ansiedeln von Deutschen in Bosnien-Herzegowina folgte in der zweiten Hälfte des 19. Jahrhunderts. Es ging in zwei Richtungen. Das erste ging aus einer privaten Initiative Ende der 60er Jahre hervor und das zweite war ein Teil der offiziellen Staatspolitik gleich nach der Okkupation Bosnien-Herzegowinas durch die österreichisch-ungarische Monarchie 1878.

\section{DAS ANSIEDELN DEUTSCHER KOLONISTEN IN BOSNIEN- HERZEGOWINA}

Eine bedeutende Rolle im Ansiedeln deutscher Kolonisten in Bosnien-Herzegowina spielte der Trappistenpfarrer Franz Pfanner. Er kam 1869 aus dem deutschen Kloster Maria-Wald bei Heimbach (Rheinland) in die Umgebung von Banja Luka. In seiner Begleitung war eine kleine Gruppe von Mönchen. Gleich nach der Ankunft fing Franz Pfanner mit der Gründung eines Trappistenklosters an. Das Kloster Maria-Stern wurde offiziell 1870 gegründet (Preradović u. a. 2017: 16). Durch hohen persönlichen Einsatz und großes Organisationsgeschick von Pfanner und der anderen Mönche und Helfer wurden „ganz erstaunliche wirtschaftliche Unternehmungen“ aufgebaut und das Kloster weitete sich aus (Westheider 2015: 125). Auch die Anzahl der Mönche wuchs schnell. Im Jahr 1882 gab es 105 Mönche. In der Zwischenzeit wurde ein größeres Klostergebäude, eine Kirche, eine Brauerei, eine Käserei, eine Ziegelei, eine Spinnerei, eine Tuchwalkerei und eine Dampfmühle aufgebaut. Im Jahr 1878 wurden ein Waisenhaus und 1878/79 ein Krankenhaus errichtet. Anfang des 20. Jahrhunderts bauten die Trappisten ein Wasserkraftwerk auf, welches das ganze Kloster und die Stadt Banja Luka mit Strom versorgte (Lamers 1970: 17, 18). Dadurch wurde das Trappistenkloster der Mittelpunkt der ganzen Region. In einem armen und unterentwickelten Land, wie es Bosnien-Herzegowina damals war, wirkte das Kloster Maria-Stern auf einen Beobachter wie ,eine Oase in der Wüste, eine wahre Burg der Zivilisation in einem Land ohne Kultur" (Strauß 1884, zit. bei Lamers 1970: 19). Die Trappisten können als Vorläufer eines massenhaften Kommens von Ausländern in die Umgebung von Banja Luka betrachtet werden (Preradović u. a. 2017: 15). 
Etwa zur Zeit der Okkupation 1878 schrieb Franz Pfanner den Artikel über Bosnien „Bosnien, ein Land für Ansiedlung“, der in der Kirchenzeitschrift „Christlicher Pilger“ in Deutschland veröffentlicht wurde. Damals war die Situation in Westdeutschland wegen eines religiösen Konflikts zwischen Katholiken und Protestanten, einer Landwirtschaftskrise, des Kulturkampfes und ungünstiger Migrationen nach Amerika sehr schwer. Deswegen wurde Pfanners Aufruf gleich aufgenommen (Malinović, Okilj, Preradović 2015: 22).

Die erste Gruppe von Ansiedlern kam 1879/80 aus Essen und der Umgebung. Es waren 25 Familien mit 196 Mitgliedern. Mit eigenen Geldmitteln kauften sie sich Grundstücke in der Umgebung von Gradiška und siedelten sich als freie Bauern an (Zaplata 1934: 157). Dieser Gruppe schlossen sich später Ansiedler aus Hannover, Oldenburg und Braunschweig an und so wurde Windthorst ${ }^{1}$, die größte deutsche Kolonie in Bosnien-Herzegowina, gegründet, die 1881 auf Antrag ihrer Einwohner als eigenständige Gemeinde organisiert wurde (Kraljačić 1989: 116). Die Kolonie bestand aus drei Teilen: zuerst entstand Unterwindthorst (meistens mit Ansiedlern aus Essen), und danach Mittelund Oberwindthorst, wo sich Familien aus verschiedenen deutschen Provinzen sowie aus Ungarn und Holland ansiedelten (Kasumović 2015: 86). Im Jahr 1885 hatte Windthorst schon 715 Einwohner (Mikić 1982: 103), während der Volkszählung 1895 waren es 1189 (Lužija 2013: 73) und im Jahr 1912 sogar 1650 (Stimmen 1912: 23).

Gleich nach der Gründung von Windthorst (heute Nova Topola) entstand 1880 auch durch die private Initiative etwa in der Mitte zwischen Banja Luka und Gradiška die zweite deutsche Kolonie, die von den Ansiedlern aus Schlesien, Hannover und Oldenburg gegründet wurde. Diese Kolonie gehörte zum Kreis Banja Luka. Am Anfang gab es 19 Familien mit 86 Mitgliedern. Im Jahr 1885 wuchs die Zahl der Mitglieder auf 306 (Hauptmann 1965: 158; Mikić 1982: 103, 104) und 1895 bei der Volkszählung auf 428 (Lužija 2013: 73). Im Jahr 1912 hatte die Kolonie 600 Einwohner (Stimmen 1912: 23). Zunächst hieß die Kolonie Maglaj am Vrbas und 1888, nach dem Besuch des Thronfolgers Rudolf, wurde sie in Rudolfstal (heute Aleksandrovac) umbenannt (Kasumović 2015: 86). Durch stets neue Zuwanderungen aus Deutschland vergrößerten sich Windthorst und Rudolfstal von Jahr zu Jahr (Stimmen 1912: 23). Die Einwohner der beiden Kolonien waren katholisch.

Eine weitere große deutsche Kolonie wurde 1886 in der Nähe von Bijeljina gegründet und hieß Franz Josefsfeld (heute Novo Selo) (Kasumović 2009: 84). Es gab etwa 100 deutsche Familien, die aus Srem kamen. Vorfahren dieser Deutschen waren noch im 18. Jahrhundert aus Baden-Württemberg gekommen und hatten sich in der Vojvodina niedergelassen (Malinović, Okilj, Preradović 2015: 31). Die Einwohner dieser Kolonie waren evangelisch. Im Jahr 1895, nach der Volkszählung, hatte diese Kolonie 1025 Einwohner (Lužija 2013: 73) und 1909 sogar 1200 (Heimfelsen 1911: 17).

Neben diesen drei Kolonien wurden noch sechs freie deutsche Kolonien gegründet, deren Einwohner mit eigenen Mitteln Grundstücke kauften, auf denen sie sich ansiedelten (Lužija 2013: 34).

1 Die Kolonie wurde nach Ludwig Windthorst benannt, der Abgeordneter im Parlament in Hannover, Justizminister in der Regierung von Hannover und später Reichstagsabgeordneter der Zentrumpartei war (Kasumović 2015: 86). 


\section{KOLONISATION ALS OFFIZIELLE STAATSPOLITIK}

Die zweite Art der Ansiedlung Bosnien-Herzegowinas durch Ausländer hatte einen offiziellen Charakter und wurde von Staatsbehörden unterstützt.

Um die Macht aufzubauen und die Wirtschaft zu fördern, siedelte das österreichisch-ungarische Regime Beamte, Soldaten und Fachleute sowie Bauern in BosnienHerzegowina an. Die ersten siedelten sich in Städten an, während die Bauern sich in Dörfern niederließen, um den Boden zu bearbeiten (Kožar 2015: 176, 177).

Ein Jahr nach der Okkupation führte die Landesregierung an, dass es das ,größte Bedürfnis" sei, ausländische Arbeitskräfte zur Förderung der Landwirtschaft und Industrie zu bekommen und dass man mit der Kolonisation beginnen könne, wenn auch nicht in großem Maße. Deswegen wurden 1880 „Verordnungen über die Kolonisation in Bosnien-Herzegowina“" erlassen. Ziel war es, ausländische Bauern, die durch das eigene Beispiel einheimischen Bauern zeigen sollten, wie man die landwirtschaftliche Produktion fördern kann, ins Land anzuziehen. Sie sollten gleichzeitig als zuverlässige Unterstützung der Okkupationsverwaltung dienen (Kraljačić 1989: 113). Durch die Kolonisation verstärkte die Okkupationsverwaltung die Zahl ihrer treuen Untertanen (Jeremić 1929, zit. bei Mikić 1982: 102). Kraljačić führt eine Initiative von Kallay² an, in der Kallay sagt, dass man durch die Ansiedlung neben ,wirtschaftlichen auch politische Ziele“ erreichen solle, indem man „das andere Element unter die serbische Bevölkerung verteile“. Auf diese Weise würden im Land starke Stützpunkte ausländischer und politisch treuer Bevölkerung gebildet und die Gebiete, die meistens von der serbischen Bevölkerung angesiedelt waren, voneinander getrennt werden (Kraljačić 1989: 115). Damit würde jeder Aufstand der Österreich-Ungarn gegenüber illoyaler Bevölkerung geschlagen werden (Preradović u. a. 2017: 14).

Die Ansiedlung konnte nur jenen Ausländern genehmigt werden, die über ein bestimmtes Kapital verfügten und die Arbeitsfähigkeit, Ausdauer und Energie hatten. Außerdem mussten sie unbescholten sein und sich in der Landwirtschaft auskennen (Kasumović 2009: 89).

Aber die ungeregelte Agrarfrage im Land verhinderte am Anfang eine erfolgreiche Durchführung der Kolonisation. Die Lösung dieses Problems war nicht leicht und dauerte lange und inzwischen vermehrte sich die Anzahl von Anträgen auf Einwanderung. Deswegen entschied die Landesregierung 1892, einen bestimmten Teil von Grundstücken zur Verfügung zu stellen und damit jenen entgegenzukommen, die sich für die Ansiedlung in Bosnien-Herzegowina interessierten. Davon zeugen die von den deutschen Bauern angesiedelten Kolonien, die von 1890 bis 1905 entstanden sind (Kasumović 2009: 87). In diesem Zeitraum entstanden 11 deutsche Kolonien (s. Auszug 1913): ${ }^{3}$ Branjevo (Zvornik 1890), Dugo Polje (Zvornik 1890), Dubrava-Königsfeld (Gradiška 1894), Vrbaška (Gradiška 1894), Prosara (Bosanska Dubica 1894),

2 Benjamin Kallay war der gemeinsame Finanzminister der österreichisch-ungarischen Monarchie von 1882 bis 1903 und gleichzeitig Landeschef von Bosnien-Herzegowina.

3 In den Klammern sind die Kreise, wo sich die Kolonien befanden, und die Gründungsjahre angeführt. 
Glogovac-Schutzberg (Prnjavor 1894-1901), Vranovac (Bosanska Dubica 1895), Kadar (Derventa 1896-1898), Nove Ularice (Tešanj 1899), Šibovska (Prnjavor 19001902), Vrbovac (Derventa 1902). Hier handelt es sich um Kolonien, die ausschließlich einen landwirtschaftlichen Charakter hatten.

Die meisten dieser Kolonien entstanden im nordwestlichen Bosnien-Herzegowina, wo es eine Menge an ungenutztem Boden gab (Hauptmann 1965: 152), der dazu noch sehr fruchtbar war. In diesen Kolonien wurden nicht nur Familien aus Deutschland angesiedelt, sondern es gab auch deutsche Familien aus anderen Regionen und Ländern, wie das südliche Ungarn, Slawonien, die Bukowina, Russland und Galizien (Kasumović 2009: 92, 93).

Neben Deutschen siedelten sich in Bosnien-Herzegowina auch Angehörige anderer Nationen, wie Italiener, Polen, Tschechen, Rutenen usw. an. Nach einer gewissen Zeit verfügte die Landesregierung nicht mehr über freie Grundstücke, die sie den Kolonisten zur Verfügung stellen konnte, sodass die offizielle Ansiedlung von Kolonisten 1905 beendet wurde, weil die Regierung keine Grundstücke mehr verteilte. Abschließend mit diesem Jahr siedelten sich in Bosnien-Herzegowina 298 deutsche Familien mit 1669 Mitgliedern an (Kasumović 2009: 93), während Kraljačić 331 deutsche Familien anführt (Kraljačić 1989: 120). Nach den Angaben aus dem Jahr 1913 wurden in Bosnien-Herzegowina 38 Kolonien auf staatlichen Grundstücken gegründet, davon 11 deutsche (Kraljačić 1989: 120). Heimfelsen (1911: 9) spricht von $19^{4}$ größeren und kleineren deutschen Kolonialgruppen, die sich auf dem Raum von der Drina im Osten, der Save entlang im Norden bis zur Una im Westen befanden. Das bedeutet, dass die deutschen Ansiedler im nordöstlichen, nördlichen und nordwestlichen Bosnien-Herzegowina lebten. Die deutschen Bauern gründeten meistens geschlossene Ansiedlungen. Es gab aber Gruppen, die sich in schon bestehenden Orten der einheimischen Bevölkerung ansiedelten. Nach den Angaben desselben Autors lebten 1911 im nordöstlichen und nördlichen Bosnien-Herzegowina etwa 8000 Deutsche. ${ }^{5}$

Obwohl die offizielle Ansiedlung ausländischer Kolonisten 1905 beendet wurde, wurde damit der Zustrom von Siedlern nicht gestoppt (Todorović Bilić 2011: 129). Aber die Landesregierung kam keinem Antragsteller entgegen, der sich in Bosnien-Herzegowina auf staatlichem Boden ansiedeln wollte (Kasumović 2009: 114). Als der wahre Grund, warum die Ansiedlung ausländischer Kolonisten beendet wurde, wird die Unzufriedenheit der einheimischen Bevölkerung wegen der Verteilung von Grundstücken an Ausländer angeführt. Die Muslime bezeichneten es als Wegnahme des staatlichen Bodens, während die serbische autonome Bewegung verlangte, den Boden an die einheimischen armen Bauern zu verteilen. Damit wurde gemeint, dass der Deutsche als Ausländer und fremder Staatsangehöriger den besten Boden besaß und reiche Ernten davon einbrachte, vom Staat Hilfe beim Straßenbau, bei der Anschaffung von Vieh und Maschinen bekam, während die einheimische Bevölkerung sich quälte zu überleben (Mikić 1982: 103). Die Kolonisation wurde auch als Mittel der Entnationalisierung betrachtet. Auch der deutsche

4 Aus dem oben angeführten Text geht hervor, dass es 20 Kolonien gab.

5 Nach der Volkszählung 1910 lebten in Bosnien-Herzegowina 22968 Deutsche (Kožar 2015: 178; Scheuringer 2019: 38). Fast ein Drittel davon machten die deutschen Bauern-Kolonisten aus. 
Konsul berichtete in Sarajevo, dass die Ansiedlung ausländischer, besonders deutscher und ungarischer Kolonisten in Bosnien-Herzegowina ,ein Dorn im Auge der einheimischen, besonders der serbischen Medien“"war (Kraljačić 1989: 119, 120). All das beeinflusste die Politik der Kolonisierung sowie deren Umfang.

\section{ORGANISATION DES LEBENS IN KOLONIEN}

Die Kolonisten, die sich in Bosnien-Herzegowina ansiedelten, hatten kein großes Vermögen. Das waren meistens Leute, die aus Not ihre ursprüngliche Heimat verließen und auf der Suche nach einem besseren Leben nach Bosnien-Herzegowina kamen. Deswegen hatten sie anfangs große Probleme bei der Organisation des Lebens. Das bezieht sich vor allem auf die Privatkolonisten in den deutschen Kolonien im nordwestlichen Bosnien. Mit eigenen Geldmitteln kauften sie Grundstücke und bauten Häuser, weswegen sie danach wenige oder gar keine Mittel mehr hatten. Die Häuser waren klein und von schlechter Qualität, sodass sie Naturereignissen nicht standhalten konnten. In manchen Häusern waren Familienzimmer, Gästezimmer, Küche, Werkstatt und Stall ein und dasselbe (Stimmen 1912: 26).

Ein paar Monate nach der Ankunft wandten sich die deutschen Ansiedler aus Essen an die Landesregierung wegen finanzieller Unterstützung. Die Landesregierung meinte aber, dass man den Privatkolonisten nicht helfen solle. Sie war sich aber bewusst, dass es sich um die ersten Kolonisten und die erste Kolonie handelte und dass deren Untergang die weitere Kolonisation negativ beeinflussen könnte (Kasumović 2015: 86).

Die ersten zwei Jahre, 1880 und 1881, waren für die Kolonisten aus Windthorst und Rudolfstal besonders schwer wegen einer schlechten Ernte und Naturereignisse, aber auch wegen der Tatsache, dass sie sich durch eine private Initiative angesiedelt hatten, weswegen sie sich selbst überlassen wurden. Außer Problemen mit der Regelung des Grundbesitzes, mit Hausbau und Steuern hatten die beiden Kolonien auch Probleme mit Gemeindegründung, Kirchenbau, Schuleröffnung, Gründung von Landwirtschaftsgenossenschaften und -gemeinschaften und Straßenbau (Mikić 1990: 189). Wegen all dieser Schwierigkeiten wandten sich die Kolonisten an die Landesregierung mit Anträgen auf Steuerabzug sowie Darlehen und Kredite. Diesen Anforderungen kam die offizielle Verwaltung nur ab und zu und symbolisch entgegen (Kasumović 2015: 88, 89). In einigen Quellen kann man aber die Angabe finden, dass die Regierung den Kolonisten aus Propagandagründen entgegenkam (Hauptmann 1965: 158) und einigen Kolonisten Holz für den Bau von Häusern und anderen Objekten, kostenloses Saatgut und finanzielle Unterstützung gab, Steuern senkte oder sie völlig vom Steuerzahlen befreite, besonders in den ersten Jahren nach der Ankunft. Dank dieser Tatsache überwanden die Kolonisten in Windthorst bald ihre Anfangsschwierigkeiten und gründeten eine der reichsten deutschen Siedlungen in Bosnien-Herzegowina (Jarak 1956, zit. bei Mikić 1982: 103). Die Sorge der Regierung ermöglichte ihnen, zu einer rationalen Wirtschaft überzugehen und dadurch einheimischen Bauern Vorbild zu sein, wie man durch die Einführung von Dampfmaschinen den Boden bearbeitet und wie man eine fortschrittliche Viehzucht betreibt. Die Kolonisten trugen auch dazu bei, dass dieses Gebiet große Menge Getreide ausführte (Mikić 1990: 189, 190). 
Die Kolonisten, die sich mit Hilfe der offiziellen Verwaltung ansiedelten, waren in einer günstigeren Lage als die Privatkolonisten, besonders diejenigen, die im Zeitraum von 1890 bis 1905 nach Bosnien-Herzegowina kamen. Die wichtigste Hilfe war die Zuteilung des kostenlosen staatlichen Bodens, den sie nach Ablauf von zehn Jahren ohne Entgelt in Besitz nehmen konnten, wenn sie einige Bedingungen erfüllten, gute Landwirtschaftler waren (Mikić 1990: 187) und den Boden gut und produktiv bearbeiteten. Außerdem gab ihnen die Regierung kostenlos Holz für den Bau von Häusern und anderen Objekten und bei schlechten Ernten zog sie von ihnen keine Steuer ein, gab ihnen zinsenlose Geldunterstützung u. ä. (Grđić 1912: 372; Mikić 1982: 105).

\section{DER WIRTSCHAFTLICHE FORTSCHRITT DEUTSCHER KOLONIEN}

Obwohl die deutschen Kolonien am Anfang mit großen Problemen konfrontiert wurden, gelang es ihnen jedoch, eine beneidenswerte wirtschaftliche Entwicklung zu erreichen. Dank ihrem Fleiß und den Erleichterungen und Unterstützungen seitens der bosnisch-herzegowinischen Regierung organisierten die Kolonisten vorbildliche Landwirtschaftsbetriebe und eine moderne Landwirtschaftsproduktion. Sogar Pächter konnten in relativ kurzer Zeit genug Geld für die Gründung eigener Bauernhöfe erwerben (Kraljačić 1989: 121).

In den größten deutschen Kolonien Windthorst und Rudolfstal benutzten die Kolonisten moderne landwirtschaftliche Maschinen: Sämaschinen, Mähmaschinen, Selbstbinder und Getreidedampfdreschmaschinen. Sie verwendeten auch Düngemittel. Weiterhin hatten sie gute Rassen von Kühen und Pferden. In den Siedlungen gab es auch Dampfmühlen, Ziegeleien, Sägewerke sowie verschiedene Handwerker (Stimmen 1912: 25). Die Situation in Franz Josefsfeld war ähnlich.

Die deutschen Kolonisten waren Pioniere im Anbau neuer Pflanzen, wie z. B. Zuckerrübe (Kraljačić 1989: 122). Die Ansiedler in Rudolfstal begannen als erste Kartoffeln anzupflanzen (Heimfelsen 1911: 55). Die Kolonisten organisierten die Produktion besonders jener Artikel, die bei der aus der Monarchie angesiedelten Stadtbevölkerung gut ankamen. So wurden in Rudolfstal Milchprodukte hergestellt, die nach Banja Luka transportiert wurden. Das Kloster Maria-Stern kaufte von dieser Kolonie Weizen und Milch ab (Kasumović 2015: 93). Die Ansiedler in Windthorst und Rudolfstal hoben sich besonders in der Produktion verschiedener Getreidesorten hervor und hatten sehr gute Ernten, sodass sie diese Produkte ausführten. Wenn sie bessere Straßenverbindungen und eine Bahn gehabt hätten, die ihnen lange versprochen wurde, hätten sie noch mehr produziert. Entfernte Märkte, besonders jene in Slawonien, blieben ihnen deswegen unzugänglich, weswegen sie einige Produkte, besonders Gemüse, nur für sich selbst produzierten (Heimfelsen 1911: 56, 62). In Windthorst wurde 1893 ein landwirtschaftlicher Verein gegründet (Kasumović 2015: 92). Wegen der erfolgreichen landwirtschaftlichen Produktion bezahlten die Kolonisten dem Staat hohe Steuern.

Um weitere landwirtschaftliche Fortschritte zu machen und günstige Darlehen und Kredite zu bekommen, verlangten die deutschen Kolonisten die Gründung von Raiffeisenkassen wie in Deutschland. Im Jahr 1912 wurden fünf Raiffeisenkassen mit 316 Mitgliedern eröffnet und im Jahr darauf gab es zehn Raiffeisenkassen mit 716 Mitgliedern. 
In einigen deutschen Kolonien wurden Milchgenossenschaften gegründet, während in Banja Luka eine Gesellschaft unter dem Namen „Milch- und Lebensmittelgesellschaft“ gegründet wurde (Kasumović 2009: 118).

Obwohl die deutschen Kolonisten einen großen Erfolg in der landwirtschaftlichen Produktion erreichten, begannen sie mit der Gründung von Bauerngenossenschaften erst im Jahr 1912. Im Jahr darauf gründeten sie den Verband deutscher bäuerlicher Kredit- und Wirtschaftsgenossenschaften in Bosnien-Herzegowina mit dem Sitz in Banja Luka (Jarak 1956, zit. bei Todorović Bilić 2011: 130).

Die Landesregierung hob Windthorst und Rudolfstal oft als erfolgreiche Kolonien hervor, die der einheimischen Bevölkerung als Vorbild dienen konnten (Kasumović 2015: 84). Was für einen Erfolg diese Kolonien erreichten, kann man aus dem folgenden Zitat herauslesen: „Das Vrbastal ist durch die Trappisten und die deutschen Kolonien Windthorst und Rudolfstal das am besten gepflegte und wirtschaftlich blühendste Stück Bosniens geworden“ (Stimmen 1912: 31).

\section{ORGANISATION DES SCHUL- UND KULTURLEBENS}

Obwohl die deutschen Kolonisten in Windthorst und Rudolfstal am Anfang auf zahlreiche Probleme und Schwierigkeiten stießen, waren sie sich jedoch bewusst, dass ihre Kinder auch unter solchen Verhältnissen eine Ausbildung brauchen. Auch da stießen sie auf Schwierigkeiten. Es mangelte an qualifizierten Lehrkräften und an einer Unterkunft für sie. Weiterhin gab es keine Schulgebäude. Aber die Kolonisten fanden eine Lösung und nutzten mal dieses mal jenes Privathaus als Schulgebäude. Am Anfang gaben Privatpersonen Unterricht. Später übernahmen den Unterricht die Schwestern vom kostbaren Blute Jesu, die Pater Franz Pfanner nach Bosnien gebracht hatte. Die Schwestern hatten ihr Kloster Nazareth in der Nähe von Banja Luka und sie kümmerten sich um die Ausbildung der Kinder dieser Kolonien (Westheider 2015: 127).

In Windthorst gab es bis 1882 keinen Unterricht. Das Haus für eine vierklassige Schule wurde 1888/89 aufgebaut. Begabte Jungen wurden nach dem Abschluss der Grundschule ins Jesuitenkollegium nach Travnik zur weiteren Ausbildung geschickt (Renner 1896: 449). Im Jahr 1911 gab es in dieser Kolonie etwa 230 Schüler (Heimfelsen 1911: 66). Im selben Jahr gab es in Rudolfstal etwa 100 Schüler, die ebenfalls eine eigene Schule hatten (Heimfelsen 1911: 57). In diesen Schulen war Deutsch die Unterrichtssprache. Damals überlegten sich die beiden Kolonien, eine weiterbildende Schule für landwirtschaftliche Kurse einzurichten (Stimmen 1912: 23). Auch Oehler ${ }^{6}$ (1918: 11, 12) betont, dass die deutschen Ansiedlungen Fortbildungsschulen bräuchten, wo Jungen einen theoretischen Unterricht über landwirtschaftliche Fragen und sonstige Fächer haben könnten, welche für den Landwirt besonders wichtig seien.

Noch einige der oben genannten deutschen Kolonien hatten ihre Schulen mit deutscher Unterrichtssprache: Dubrava-Königsfeld, Franz Josefsfeld, Vrbaška, GlogovacSchutzberg und Vranovac. In einigen Ansiedlungen gab es Schulen mit einer deutschen

6 W. J. Oehler war Pfarrer der autonomen evangelischen Westgemeinden im Kreis Banja Luka und Bihać und stellvertretender Direktor des Verbandes deutscher bäuerlicher Kredit- und Wirtschaftsgenossenschaften in Bosnien. 
und einer serbokroatischen Abteilung: Branjevo, Kadar, Nove Ularice, Šibovska. In den anderen Kolonien gab es entweder keine Schule oder ein unqualifizierter Lehrer hielt den Unterricht (Auszug 1913), obwohl Kasumović (2009: 117) anführt, dass es 1911 in Bosnien-Herzegowina 36 Schulen oder Schulabteilungen gab, wo der Unterricht in deutscher Sprache abgehalten wurde.

Im Jahr 1911 erließ das bosnische Parlament ein Gesetz, in dem geregelt wurde, dass den deutschen Schulen keine finanziellen Mittel zur Verfügung gestellt werden. Ein Jahr zuvor erließ das Parlament eine Resolution, nach der die Landessprache die Unterrichtssprache in allen Schulen sein sollte, die von der Landesregierung finanziert oder subventioniert wurden (Kasumović 2009: 117). Es gab 12 deutsche Schulen, die bis 1910 eine finanzielle Unterstützung vom Staat bekamen. Der Regierung wurde vorgeworfen, dass sie sich mehr um die Schulen der Kolonisten kümmere als um die einheimischen Schulen und dass deutsche Kinder die deutsche Geschichte lernen dürften, während die Geschichte der Serben in den Schulen verboten werde (Grđić 1912: 378, 379). Obwohl die Einführung der serbokroatischen Sprache die Bedingung für die Hilfeleistung wurde, hielten diese Schulen an Deutsch als Unterrichtssprache fest und bekamen Hilfe von deutschen Stiftungen und Kirchen (Richembergh 2010: 102). Auch Grđić (1912: 379) führt an, dass in einigen Schulen der Unterricht weiterhin auf Deutsch abgehalten wurde, was man auch dem „Auszug“ (1913) entnehmen kann.

Bei der Bewahrung der Nationalidentität der deutschen Kolonisten half auch die Organisation des Kirchenlebens. Wie die Schulen wurden am Anfang auch die Kirchen in Privathäusern untergebracht. Später wurden in allen großen deutschen Kolonien Kirchen gebaut. Laut Heimfelsen (1911: 61) wurde in Mittel-Windthorst die schönste gotische Kirche in Bosnien-Herzegowina errichtet. Sie wurde 1891 und die Kirche in Rudolfstal 1895/96 errichtet. Darum kümmerten sich meistens die Mönche aus dem Trappistenkloster Maria-Stern und Hilfe kam auch aus Deutschland (Stimmen 1912: 29).

Im Jahr 1906 wurde die Gesellschaft der Deutschen mit dem Sitz in Sarajevo gegründet. Die Gesellschaft führte ihre Aktivitäten in allen deutschen Kolonien mit Hilfe von Filialen durch. Sie setzte sich für die Bewahrung der deutschen Lebensweise und der Schulangelegenheiten ein. Pfarrer Oehler gründete vor dem Ersten Weltkrieg eine Lesegesellschaft, eine Wanderbibliothek und eine Fotozentrale (Kasumović 2009: 118).

$\mathrm{Da}$ die Ansiedler meistens isolierte und geschlossene Siedlungen gründeten, konnten sie ihre ethnische Reinheit bewahren und ihr eigenes soziales und kulturelles Leben organisieren, das auf den aus der Heimat mitgebrachten Werten (sie bauten Häuser nach dem Vorbild der ehemaligen Heimat, sie zogen sich genauso wie in der ehemaligen Heimat an, sie behielten ihren Dialekt) basierte. Außerdem trugen die größten deutschen Siedlungen deutsche Namen (vgl. Radanović 2018: 337--349). Dadurch bewahrten sie ihre Nationalidentität und schützten sich vor Assimilation (Kraljačić 1989: 122).

\section{SCHLUSSFOLGERUNGEN}

Die oben angeführten Erkenntnisse zahlreicher Autoren, die Archivquellen benutzten oder Zeitgenossen des betrachteten Zeitraums und der genannten Ereignisse waren, geben Einblick ins Leben eines Teiles der deutschen Nationalminderheit in 
Bosnien-Herzegowina Ende des 19. und Anfang des 20. Jahrhunderts. Die deutschen Bauern, die teils aus Deutschland teils aus anderen Gebieten Europas nach BosnienHerzegowina kamen, begannen bald das Leben nach dem Vorbild der ursprünglichen Heimat zu organisieren, obwohl sie am Anfang auf Schwierigkeiten stießen. Dank ihrer Energie, Ausdauer und unermüdlichen Arbeit, aber auch der Hilfe, die sie von den bosnisch-herzegowinischen Behörden bekamen, gelang es ihnen, vorbildliche Siedlungen, besonders im nordwestlichen Teil Bosnien-Herzegowinas, zu bilden. Die rationale und fortschrittliche landwirtschaftliche Produktion brachte bald gute Ergebnisse, sodass ihre Ernten viel reicher waren als die der einheimischen Bevölkerung. All das ermöglichte die Organisation des sozialen, kulturellen und kirchlichen Lebens und die Bildung von meist geschlossenen und isolierten Siedlungen ermöglichte die Bewahrung des Nationalwesens, der Kultur, der Sitten und der Sprache.

Da Bosnien-Herzegowina damals mit der ungeregelten Agrarfrage, feudalen Verhältnissen und mit einer äußerst ländlichen Bevölkerung unterentwickelt war, war es für die deutschen Siedlungen nicht so schwer „eine Oase in der Wüste“ oder „eine wahre Burg der Zivilisation in einem Land ohne Kultur" zu sein. Schließlich kämpfte die arme einheimische Bevölkerung um das Überleben und hatte weder Zeit noch Möglichkeit, sich mit der Kultur zu beschäftigen, wie das das reichere Westeuropa tat. Trotzdem möchte man den deutschen Bauern ihren Erfolg nicht absprechen.

\section{Literatur}

Auszug aus der Reiserelation des Kreisvorstehers Ladislaus Baron über Kolonien in Bosnien. (1913). ABH Sarajevo zl 15.514 vom 19. Februar 1913.

BETHKE, Carl (2015) „Die Zeitungen „Bosnische Post“ und „Sarajevoer Tagblatt", 1903-1913.“'In: E. S. Omerović (Hrsg.), 137-174.

BILIĆ TODOROVIĆ, Marijana (2011) „Austrougarska kolonizacija u Bosni i Hercegovini.“ Glasnik Udruženja arhivskih radnika Republike Srpske. III, 3. Banja Luka, 119-135.

GRĐIĆ, Šćepan (1912) „Kolonizacija u Bosni i Hercegovini.“ Pregled. Časopis za nauku i socijalni život. Sarajevo, 01.10.1912, 367-382.

HAUPTMANN, Ferdo (1965) „Reguliranje zemljišnog posjeda u Bosni i Hercegovini i počeci naseljavanja stranih seljaka u doba austrougarske vladavine." Godišnjak društva istoričara Bosne i Hercegovine. Sarajevo, XVI, 151-171.

HEIMFELSEN, Josef (1911) Die deutschen Kolonien in Bosnien. Sarajevo.

KASUMOVIĆ, Amila (2009) „Modaliteti eksterne kolonizacije u Bosni 1890-1914: case study za njemačke erarne kolonije.“ Prilozi, 38. 81-120.

KASUMOVIĆ, Amila (2015) „Austrougarska kolonizaciona politika u Bosni i Hercegovini i prvi njemački doseljenici.“ In: E. S. Omerović (Hrsg.), 75-95.

KOŽAR, Azem (2015) „Doseljavanje Nijemaca-kolonista na područje kotara Tešanj u vrijeme austrougarske uprave u Bosni i Hercegovini.“ In: E. S. Omerović (Hrsg.), $175-188$.

KRALJAČIĆ, Tomislav (1989) „Kolonizacija stranih seljaka u Bosnu i Hercegovinu za vrijeme austrougarske uprave.“Istorijski časopis. XXXVI, 111-124. 
LAMERS, Friedrich G. (1970) 65 Jahre Kolonie in Bosnien in Jugoslawien. St. Gabriel/Mödling bei Wien.

LUŽIJA, STOJANKA (2013) Njemačke agrarne kolonije u Bosni i Hercegovini (1878-1914). Banja Luka: Art print.

MALINOVIĆ, Miroslav/Milijana OKILJ/Ljubiša PRERADOVIĆ (2015) „The Colonization of German National Minority and Its Sacred Architecture in Republik of Srpska. In: $A G G+, 3$. http://doisrpska.nub.rs/index.php/aggplus/article/view/2924.

MIKIĆ, Đorđe (1982) „O privrednim i socijalnim prilikama u Bosanskoj krajini u prvim godinama austrougarske okupacije (1878-95).“ Istorijski zbornik. Banja Luka, 75-110.

MIKIĆ, Đorđe (1990) „O kolonizaciji stranih seljaka u Bosni i Hercegovini u vrijeme austrougarske uprave.“ In: N. Šehić (Hrsg.), Migracije i Bosna i Hercegovina. Zbornik. Sarajevo: Institut za istoriju u Sarajevu/Institut za proučavanje nacionalnih odnosa, 181-193.

OEHLER, W. J. (1918) Die deutschen Kolonien in Bosnien. Banja Luka.

OMEROVIĆ, Enes S. (Hrsg.) (2015) Nijemci u Bosni i Hercegovini i Hrvatskoj-nova istraživanja i perspektive. Zbornik radova. Sarajevo: Institut za istoriju.

PRERADOVIĆ, Ljubiša u. a. (2017) Vjerski objekti nacionalnih manjina u Republici Srpskoj. Banja Luka: Grafid.

RADANOVIĆ, Sanja (2018) „Mehrnamigkeit in der Toponymik in Bosnien und Herzegowina 1878-1918.“ In: P. Ernst u. a. (Hrsg.) Mehrnamigkeit zwischen Sprachwissenschaft, Sprachgeschichte und Sprachpolitik. Österreichische Namenforschung, Beiheft 6, Wien: Praesens, 337-349.

RENNER, Heinrich (1896) Durch Bosnien und die Herzegowina kreuz und quer. Berlin: saxioniabuch.

RICHEMBERGH, Goran Beus (2010) Nijemci, Austrijanci i Hrvati I. Prilozi za povijest njemačko-austrijske nacionalne manjine u Hrvatskoj i Bosni i Hercegovini. Zagreb/Sarajevo: Synopsis.

SCHEURINGER, Hermann (2019) „Bosniens rudimentärster Kontakt mit dem Deutschen - Die Posavina als Nachhut der großen Schwabensiedlung.“ In: S. Mešić/H. Scheuringer (Hrsg.), Deutsch in Bosnien-Herzegowina. Sarajevo: Philosophische Fakultät der Universität Sarajevo, 30-40.

Stimmen aus Bosnien (1912). Illustrierte Blätter in zwangloser Folge. Sarajevo.

ŠEHIĆ, Nusret (1990) „Politika iseljavanja i useljavanja na prodručju Bosne i Hercegovine 1918-1921. Godine. “In: N. Šehić (Hrsg.), Migracije i Bosna i Hercegovina. Zbornik. Sarajevo: Institut za istoriju u Sarajevu/Institut za proučavanje nacionalnih odnosa Sarajevo, 195-222.

WESTHEIDER, Rolf (2015) „Von der Ems an den Vrbas: Norddeutsche in Windthorst und Rudolfstal.“ In: E. S. Omerović (Hrsg.), 123-136.

ZAPLATA, Rudolf (1934) „Postanak njemačkih naseobina kod Banje Luke.“ In: Glasnik jugoslovenskog profesorskog društva. 10-12, 918-922. 


\section{Zusammenfassung \\ DEUTSCHE KOLONIEN IN BOSNIEN-HERZEGOWINA BIS 1914}

Die Regierung der österreichisch-ungarischen Monarchie in Bosnien-Herzegowina von 1878 bis 1918 wurde unter anderem durch die Ansiedlung von Beamten, Ingenieuren, Handwerkern, Bauern und Menschen anderer Berufe aus verschiedenen Gebieten der Monarchie gekennzeichnet. Einen wichtigen Platz unter ihnen nahmen deutsche Bauern ein, welche in den letzten Jahrzehnten des 19. Jahrhunderts fruchtbare Gebiete im Norden Bosnien-Herzegowinas ansiedelten, besonders dessen nordwestliche Gebiete, und ihre Kolonien gründeten. Trotz Schwierigkeiten, auf die sie am Anfang stießen, gelang es ihnen, dank ihrer unermüdlichen Arbeit und Ausdauer, der Nutzung von für die damalige Zeit modernen landwirtschaftlichen Maschinen und Geräten und dem Unternehmen einiger Maßnahmen, sowie der Hilfe aus Deutschland und Erleichterungen, welche sie von der bosnisch-herzegowinischen Regierung bekamen, eine beträchtliche Steigerung der landwirtschaftlichen Produktion zu erreichen. Bereits in den ersten Jahren begannen sie Kirchen und Schulen zu bauen, wobei sie große Unterstützung bei den Mönchen aus dem Trappisten-Kloster in der Nähe von Banja Luka fanden. Dadurch konnten sie ihr soziales und kulturelles Leben organisieren und ihre nationale Identität bewahren. Unter den deutschen Kolonien zeichneten sich besonders Windthorst und Rudolfstal in der Nähe von Banja Luka aus, sowohl durch ihre Oberfläche und Einwohnerzahl als auch durch eine erfolgreiche landwirtschaftliche Produktion. Dank den deutschen Kolonien wurde die landwirtschaftliche Produktion in Bosnien-Herzegowina erheblich verbessert.

Schlüsselwörter: deutsche Bauern, deutsche Kolonien, österreichisch-ungarische Monarchie, das Ansiedeln, die landwirtschaftliche Entwicklung

\section{Abstract \\ GERMAN COLONIES IN BOSNIA AND HERZEGOVINA UNTIL 1914}

The Austro-Hungarian rule in Bosnia and Herzegovina (1878-1918) was marked, among other things, by the immigration of numerous officials, engineers, craftsmen, peasants and people of other professions from various parts of the Monarchy. A significant subgroup consisted of German peasants who in the last decades of $19^{\text {th }}$ century settled in the fertile lands of northern Bosnia and Herzegovina, especially its northwestern parts. Despite initial difficulties, they became highly successful and enjoyed high agricultural productivity, which was achieved through hard work, perseverance, wise planning decisions as well as the use of modern agricultural machines. There was also aid coming from Germany and support offered by the authorities in Bosnia and Herzegovina. Soon after their arrival, the peasants began to build churches and schools, whereby they were helped by a Trappist monastery near Banja Luka. As a consequence, they managed to organize their social and cultural life and preserve their national identity. Among the German colonies, Windthorst and Rudolfstal near Banja 
Luka stood out, both in terms of their size and population, and in terms of agricultural output. Thanks to the German colonies, agricultural production in Bosnia and Herzegovina improved significantly.

Keywords: German peasants, German colonies, Austro-Hungarian Monarchy, immigration, agricultural development

\section{Povzetek \\ NEMŠKE KOLONIJE V BOSNI IN HERCEGOVINI DO LETA 1914}

Vladavino avstro-ogrske monarhije v Bosni in Hercegovini od 1878 do 1918 je zaznamovalo priseljevanje uradnikov, inženirjev, obrtnikov, kmetov ter ljudi drugih poklicev iz različnih območij monarhije. Pomembno mesto med njimi imajo nemški kmetje, ki so v zadnjih desetletjih 19. stoletja naselili rodovitna območja na severu Bosne in Hercegovine, še posebej njene severozahodne predele, ter tam ustanavljali svoje kolonije. Zaradi svoje delavnosti in vzdržljivosti, uporabe za takratne čase modernih kmetijskih strojev in naprav, sprejetja nekaterih ukrepov, pa tudi pomoči iz Nemčije ter ugodnosti, ki so jih prejeli od avstro-ogrskih oblasti, jim je kljub težavam, na katere so naleteli na začetku, uspelo kmetijsko produkcijo močno povečati. Že v prvih letih so začeli graditi cerkve in šole, pri tem pa so imeli veliko podporo menihov iz samostana trapistov v bližini Banja Luke. Na ta način jim je uspelo organizirati družbeno in kulturno življenje ter ohraniti nacionalno identiteto. Med nemškimi kolonijami najbolj izstopata Windthorst (Nova Topola) in Rudolfstal (Aleksandrovac) v bližini Banja Luke, tako zaradi svoje velikosti in števila prebivalcev kot tudi zaradi uspešnega kmetijskega gospodarstva. Po zaslugi nemških kolonij je kmetijsko gospodarstvo v BiH močno napredovalo.

Ključne besede: nemški kmetje, nemške kolonije, avstro-ogrska monarhija, priseljevanje, kmetijski razvoj 